\title{
Tratamientos psicológicos para los trastornos adaptativos: una revisión
}

\author{
NOELIA SIMÓN SÁNCHEZ \\ al225816@uji.es \\ MAR MolÉs AmpostA \\ molesm@uji.es \\ SoledAd Quero CASTELLANO \\ squero@uji.es
}

\section{Resumen}

Introducción: El trastorno adaptativo (TA) es clasificado en el DSM-V en la categoría de trastornos relacionados con el trauma y factores de estrés. A pesar del cambio en la conceptualización, todavía existen problemas de diagnóstico. Paradójicamente, cuenta con una alta prevalencia, siendo de los más frecuentes en atención primaria y hospitalaria. Causa gran sufrimiento personal ya que se produce un deterioro significativo del funcionamiento social u ocupacional. Sin embargo, se observa escasez de investigaciones y ausencia de manuales de evaluación y tratamiento que lo incluyan. En cuanto a la intervención, no existen en la actualidad tratamientos psicológicos basados en la evidencia (TPBE). Método: Se realizó una búsqueda en PsycNET, ScienceDirect, PubMed y PsycARTICLES. En una primera fase, se revisaron los artículos existentes relacionados con el TA. En una segunda fase, se profundizó en los tratamientos psicológicos utilizados para el TA. Resultados: En primer lugar, se puso de manifiesto una escasez de estudios tratándose de recomendaciones generales, sugerencias e investigaciones aisladas. En cuanto a estudios específicos sobre el tratamiento psicológico, encontramos estudios de casos no controlados y 6 estudios experimentales variados y con diferentes enfoques terapéuticos, incluyendo algunos el uso de las TIC. Discusión: Es necesario desarrollar TPBE para el TA dado que los estudios encontrados presentan limitaciones metodológicas y de validez externa. Para futuras investigaciones, el uso de las TIC como tratamiento puede ser útil ya que estudios hallados hasta la fecha han demostrado ser prometedores y beneficiosos.

Palabras clave: trastornos relacionados con el trauma y el estrés, trastornos adaptativos, tratamientos psicológicos, TIC, revisión.

\section{Abstract}

Introduction: Adjustment Disorders (AD) are classified in the DSM-5 as the traumarelated disorders and stress factors category. Despite the change in conceptualisation, diagnostic problems still exist. Paradoxically, it has a high prevalence and is the 
most frequent in primary and hospital care. It causes great personal suffering given the significant impairment of social or occupational functioning. Research into it, evaluations, and treatment manuals that include it, are lacking. Currently there are no evidence-based psychological treatments (EBPT) for intervention. Method: A search in Psycnet, ScienceDirect, PubMed and PsycARTICLES was performed. Firstly, existing AD-related articles were reviewed. Secondly, we profoundly examined the psychological treatments used for AD. Results: Firstly, the search revealed a shortage of case studies of general recommendations, suggestions and isolated research. Among specific studies on psychological treatment, we found no controlled studies and six varied experimental studies with different therapeutic approaches, of which some have used ICTS. Discussion: It is necessary to develop EBPT for AD as the found studies show methodological limitations and external validity. Employing ICTs as treatment may be useful for future research since the studies found to date have being promising and beneficial.

Keywords: Trauma-related disorders and stress, adjustment disorders, psychological treatments, ICTs, review.

\section{Introducción}

Según el DSM-V (APA, 2013), el trastorno adaptativo (TA) se caracteriza por el desarrollo de síntomas emocionales y/o comportamentales en respuesta a un o varios factores de estrés identificables. Los síntomas aparecen a lo largo de los primeros 3 meses desde que se desencadena el factor y no perduran más de 6 meses. Los factores estresantes pueden ser muy variados (una ruptura sentimental, problemas económicos, independización del hogar, problemas laborales, etc.). Los síntomas son considerados clínicamente significativos cuando se trata de un malestar intenso o desproporcionado a la gravedad o intensidad del factor de estrés, teniendo en cuenta el contexto externo y los factores culturales que podrían influir en la gravedad y la presentación de los síntomas y cuando se produce un deterioro significativo en lo social, laboral u otras áreas importantes del funcionamiento.

EI TA constituye un importante problema de salud mental pública siendo uno de los trastornos más diagnosticados por psicólogos y psiquiatras. Por lo que se refiere a la prevalencia, se estima una alta tasa entre el 5-21 \% (APA, 2000; Despland, Monod y Ferrero, 1995; Fabrega, Mezzich y Mezzich, 1987) y este problema se asocia a un deterioro significativo laboral y social (Maercker, Einsle y Kollner, 2007) causante de altas tasas de bajas por enfermedad y discapacidad (van der Klink y cols., 2003). En ocasiones los síntomas son tan intensos que pueden desencadenar comportamientos autolesivos o, en el peor de los casos, el suicidio (Andreasen y Hoenk, 1982; Benton y Lynch, 2006). En este sentido, el TA es más común que la depresión mayor en pacientes hospitalizados que padecen una enfermedad grave (Silverstone, 1996; Strain y cols., 1998) y en personas que asisten al servicio de urgencias tras autolesionarse (Taggart y cols., 2006).

A pesar de la gravedad del problema, todavía es considerado una categoría residual en la cual se clasifica a pacientes que no cumplen los criterios diagnósticos para otros trastornos, como ansiedad y depresión, o como diagnóstico temporal cuando los criterios para el trastorno de estrés postraumático o un trastorno del estado de ánimo no están claros (Casey, 2011). Las limitaciones relacionadas con su evaluación y los tratamientos son también relevantes. En la 
actualidad, muchos instrumentos de detección ampliamente utilizados en la investigación no incluyen su diagnóstico (Casey y cols., 2006). Se hace notable la falta de investigación y la ausencia de manuales de evaluación y tratamiento que incluyan el trastorno. En este sentido, no existen tratamientos psicológicos basados en la evidencia (TPBE). Por lo tanto, se hace necesario dedicar esfuerzos para desarrollar TPBE que ayuden a los clínicos a reducir o eliminar este problema a través de la intervención y para mejorar la compresión en el campo del TA que tan poco estudiado ha sido hasta la fecha.

El objetivo del presente trabajo es llevar a cabo una revisión de la literatura científica sobre los tratamientos psicológicos diseñados para el TA y poner de manifiesto las limitaciones encontradas. Al mismo tiempo, se pretende sensibilizar respecto a la importancia que tiene este trastorno en nuestra sociedad, de su alta prevalencia y los serios problemas de salud que puede conllevar si no se diagnostica y se trata adecuadamente.

\section{Método}

Se realizó una búsqueda en bases de datos como PsycNet, ScienceDirect, PubMed y PsycARTICLES. En la tabla 1 se pueden ver los términos de búsqueda acotados. La revisión bibliográfica se llevó a cabo en dos fases. En una primera fase, la búsqueda se centró en revisar artículos existentes en la literatura que hablaran del TA de manera general. En una segunda fase, se realizó una búsqueda más específica de los tratamientos psicológicos utilizados para el trastorno.

Tabla 1. Bases de datos y términos de búsqueda acotados en la búsqueda bibliográfica

\begin{tabular}{|c|c|}
\hline Bases de datos & Términos de búsqueda \\
\hline $\begin{array}{l}\text { PsycnET } \\
\text { Science Direct } \\
\text { PubMed } \\
\text { PsyctARTICLES }\end{array}$ & 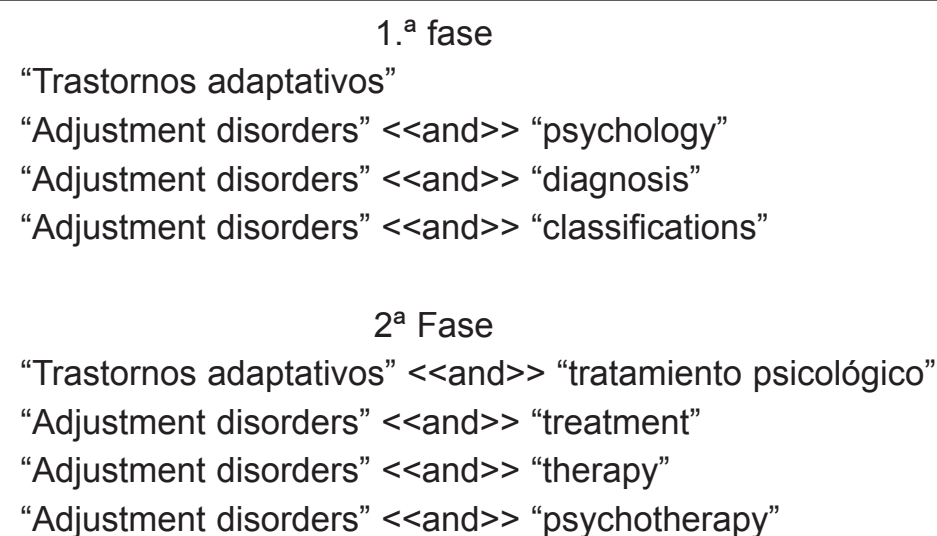 \\
\hline
\end{tabular}

Los artículos seleccionados incluían información relevante acerca del TA (prevalencia, diagnóstico, clasificación, interferencia, subtipos, etc.), así como investigaciones experimentales, cuasiexperimentales o estudios no controlados que utilizaban técnicas psicológicas para el tratamiento de los TA. La selección de los estudios se realizó siguiendo los criterios mencionados para explorar más ampliamente la literatura. 


\section{Resultados}

Lo que se encuentra en la literatura son, en primer lugar, recomendaciones generales y, en segundo lugar, un número reducido de estudios en los que se pone a prueba un determinado tratamiento para el abordaje de este problema.

Atendiendo a las recomendaciones generales, Casey (2009) propone tres componentes principales que agrupan lo que otros autores (Andrews, 1995, Benton y Lynch, 2006; Strain, 1995; Strain y Diefenacher, 2008; Strain, Klipstein y Newcorn, 2010) también consideran relevante a la hora de basar las intervenciones psicológicas para los TA: 1) reducir o eliminar el evento estresante tomando medidas como la técnica de solución de problemas; 2) facilitar la adaptación a través de medidas que se dirigen a eventos estresantes crónicos que no pueden ser eliminados (enfermedades) y que ayudan a recontextualizar la situación como la psicoeducación y la reestructuración cognitiva y, además, potenciar un sistema de apoyo que ayude a la persona a manejar mejor la situación, especialmente la ayuda práctica; 3) cambiar la respuesta emitida a través de la relajación, la verbalización de miedos y emociones, explorar el significado que tiene el evento estresante para la persona puede cambiar la respuesta emitida al suceso estresante.

En cuanto a estudios realizados sobre el tratamiento psicológico de los TA, los estudios hallados son escasos, muy variados e incluyen diferentes enfoques terapéuticos, por lo que se han clasificado según el diseño de investigación utilizado. En primer lugar, en la tabla 2 se agrupan los estudios no controlados (diferentes estudios de caso y estudios con un único grupo o series de casos). En segundo lugar, se recogen estudios experimentales entre grupos que comparan como mínimo dos condiciones experimentales (véase la tabla 3) así como aquellos que hacen uso de las tecnologías de la información y la comunicación (TIC) como tratamiento (véase la tabla 4).

Tabla 2

Estudios no controlados sobre tratamientos psicológicos para los TA

\begin{tabular}{llll}
\hline \multicolumn{1}{c}{ Autores } & \multicolumn{1}{c}{ Enfoques } & \multicolumn{1}{c}{ Técnicas } & Tipo de estudio \\
\hline $\begin{array}{l}\text { Nardi, Lichtenbert y } \\
\text { Kaplan (1994) }\end{array}$ & $\begin{array}{l}\text { Terapia congnitivo- } \\
\text { conductual (TCC) }\end{array}$ & $\begin{array}{l}\text { Desensibilización } \\
\text { Reestructuración cognitiva } \\
\text { Estrategias de afrontamiento }\end{array}$ & Series de casos \\
\hline Mihelich (2000) & $\begin{array}{l}\text { Desensibilización y } \\
\text { reproceso por el } \\
\text { movimiento de los } \\
\text { ojos (EMDR) }\end{array}$ & Estimulación bilateral ocular & Series de casos \\
\hline Frankel (2001) & $\begin{array}{l}\text { Terapia de fortale- } \\
\text { cimiento del ego }\end{array}$ & Estrategias de afrontamiento & Estudio de casos \\
\hline & & $\begin{array}{l}\text { Psicoeducación } \\
\text { Relajación autógena } \\
\text { Respiración controlada }\end{array}$ & \\
Belchi, Vilardaga y & TCC & $\begin{array}{l}\text { Reestructuración cognitiva } \\
\text { Exposición } \\
\text { Estrategias de distracción }\end{array}$ & Estudio de casos \\
& Tareas para casa & \\
\hline
\end{tabular}




\begin{tabular}{|c|c|c|c|}
\hline Autores & Enfoques & Técnicas & Tipo de estudio \\
\hline Bazarra (2004) & $\begin{array}{l}\text { Terapia psicológica } \\
\text { adyuvante (TCC) }\end{array}$ & $\begin{array}{l}\text { Relajación } \\
\text { Tareas graduadas } \\
\text { Planificación de actividades } \\
\text { Reestructuración cognitiva } \\
\text { Estrategias de afrontamiento } \\
\text { Ventilación de sentimientos }\end{array}$ & Estudio de casos \\
\hline $\begin{array}{l}\text { Powell y McCone } \\
\text { (2004) }\end{array}$ & TCC & $\begin{array}{l}\text { Control del impulso } \\
\text { Reestructuración cognitiva } \\
\text { Manejo del estrés }\end{array}$ & Estudio de casos \\
\hline $\begin{array}{l}\text { Jojic y Leposavic } \\
\text { (2005) }\end{array}$ & $\begin{array}{l}\text { Entrenamiento au- } \\
\text { tógeno }\end{array}$ & Entrenamiento autógeno & Único grupo \\
\hline Herrero y Cano (2010) & TCC & $\begin{array}{l}\text { Reestructuración cognitiva } \\
\text { Solución de problemas } \\
\text { Exposición } \\
\text { Relajación muscular progresiva } \\
\text { Respiración e imaginación }\end{array}$ & Estudio de casos \\
\hline
\end{tabular}

Tabla 3

Estudios controlados sobre tratamientos psicológicos para TA

\begin{tabular}{|c|c|c|c|}
\hline Autores & $\begin{array}{l}\text { Criterios de } \\
\text { inclusión }\end{array}$ & Grupos experimentales & Resultados \\
\hline $\begin{array}{l}\text { González- } \\
\text { Jaimes y } \\
\text { Turnbull-Plaza } \\
\text { (2003) }\end{array}$ & $\begin{array}{l}\text { Diagnóstico de } \\
\text { infarto agudo de } \\
\text { miocardio. } \\
X>55 \text { en la } \\
\text { triada depresiva } \\
\text { neurótica } \\
\text { (MMPI). } \\
\text { Diagnóstico de TA. }\end{array}$ & $\begin{array}{l}\text { GP }=\text { Gestalt }(N=36) \\
M P=\text { Terapia del espejo } \\
(N=36) \\
M C=\text { Consejo médico } \\
(N=36) \\
C G(N=36) \\
\text { Total: } 144\end{array}$ & $\begin{array}{l}\text { MP = Tratamiento eficaz en la disminu- } \\
\text { ción de la sintomatología a corto plazo. } \\
\text { Diferencias significativas entre el grupo } \\
\text { MP y los grupos restantes en el postra- } \\
\text { tamiento. Seguimiento de } 6 \text { meses: } \\
\text { disminución preocupación por la salud } \\
\text { en todos los grupos excepto cG. }\end{array}$ \\
\hline $\begin{array}{l}\text { Van der Klink, } \\
\text { Blonk, Schene } \\
\text { y Van Dijk } \\
(2003)\end{array}$ & $\begin{array}{l}\text { Trabajadores } \\
\text { que habían soli- } \\
\text { citado su prime- } \\
\text { ra baja por en- } \\
\text { fermedad como } \\
\text { consecuencia de } \\
\text { presentar el di- } \\
\text { agnóstico de TA. }\end{array}$ & $\begin{array}{l}\text { GE TCC }(\mathrm{N}=109) \\
\text { GC }(\mathrm{N}=83) \\
\text { Total: } 192\end{array}$ & $\begin{array}{l}\text { A los } 3 \text { meses, un } \text {. }^{\circ} \text { significativa- } \\
\text { mente mayor de pacientes del GE } \\
\text { habían vuelto al trabajo en comparaci- } \\
\text { ón con el GC. } \\
\text { A los } 12 \text { meses todos los pacientes } \\
\text { habían vuelto a trabajar, } \\
\text { No hubo diferencias entre los dos } \\
\text { grupos con respecto a la disminución } \\
\text { de los síntomas. }\end{array}$ \\
\hline
\end{tabular}




\begin{tabular}{|c|c|c|c|}
\hline Autores & $\begin{array}{l}\text { Criterios de } \\
\text { inclusión }\end{array}$ & Grupos experimentales & Resultados \\
\hline $\begin{array}{l}\text { Sterinhardt y } \\
\text { Dolbier (2008) }\end{array}$ & $\begin{array}{l}\text { Ser universita- } \\
\text { rio. } \\
\text { Estar atravesan- } \\
\text { do una situación } \\
\text { de elevado es- } \\
\text { trés académico. }\end{array}$ & $\begin{array}{l}\text { GE TCC }(N=30) \\
\text { GCLE }(N=27) \\
\text { Total: } 57\end{array}$ & $\begin{array}{l}\text { El GE obtuvo puntuaciones significati- } \\
\text { vamente más altas de resiliencia, es- } \\
\text { trategias de afrontamiento más efica- } \\
\text { ces, mayores puntuaciones en } \\
\text { factores de protección y puntuaciones } \\
\text { más bajas en la sintomatología des- } \\
\text { pués de la intervención que el GC. }\end{array}$ \\
\hline $\begin{array}{l}\text { Srivastava, } \\
\text { Uddip y Vivek } \\
\text { (2011) }\end{array}$ & $\begin{array}{l}\text { Diagnóstico de } \\
\text { TA. } \\
\text { No tener otro } \\
\text { trastorno psi- } \\
\text { quiátrico o co- } \\
\text { morbilidad. } \\
\text { No tomar medi- } \\
\text { cación. }\end{array}$ & $\begin{array}{l}\text { GE }(N=15) \text { Meditación } \\
\text { del yoga. } \\
\text { GC }(N=15)=\text { Asesora- } \\
\text { miento general. } \\
\text { Total: } 30\end{array}$ & $\begin{array}{l}\text { Se produjo un cambio gradual en } \\
\text { todos los parámetros de evaluación } \\
\text { seleccionados en ambos grupos. Pero } \\
\text { fueron más significativos en el GE. }\end{array}$ \\
\hline $\begin{array}{l}\text { Ben-Itzhak y } \\
\text { cols. (2012) }\end{array}$ & $\begin{array}{l}\text { Diagnóstico de } \\
\text { TA. } \\
\text { Tener de } 20 \text { a } \\
65 \text { años. } \\
\text { Que hablen } \\
\text { hebreo. }\end{array}$ & $\begin{array}{l}\text { GE grupo con psicotera- } \\
\text { pia breve }(\mathrm{N}=48) \\
\text { GE con psicoterapia } \\
\text { intermedia }(\mathrm{N}=43) \\
\text { Total: } 91\end{array}$ & $\begin{array}{l}\text { Mejoría en ambos grupos. La psicote- } \\
\text { rapia breve se mostró tan eficaz como } \\
\text { la psicoterapia intermedia tanto al final } \\
\text { del tratamiento como durante el segui- } \\
\text { miento. }\end{array}$ \\
\hline $\begin{array}{l}\text { Hsiao y cols. } \\
(2014)\end{array}$ & $\begin{array}{l}\text { Tener entre } 18 \text { y } \\
65 \text { años. } \\
\text { Tener un diag- } \\
\text { nóstico de TA } \\
\text { con depresión } \\
\text { según la MINI y } \\
\text { el DSM-IV. }\end{array}$ & $\begin{array}{l}\text { GE BMS (Body-Mind- } \\
\text { Spirit) }(N=21) \\
\text { GC }(N=18) \\
\text { Total: } 39\end{array}$ & $\begin{array}{l}\text { No se apreciaron diferencias entre el } \\
\text { GE BMS y GC en cuanto a sintomatolo- } \\
\text { gía depresiva o ansiosa. Respecto a } \\
\text { la ideación suicida, se vio reducida en } \\
\text { GE BMS. Los cambios producidos en } \\
\text { los patrones diurnos de cortisol tam- } \\
\text { bién fueron significativamente diferen- } \\
\text { tes, mostrándose a favor del GE BMS. }\end{array}$ \\
\hline
\end{tabular}

Tabla 4

Estudios que utilizan las TIC como tratamientos psicológicos para los TA

\begin{tabular}{lccc}
\hline \multicolumn{1}{c}{ Autores } & Año & TIC & Tipo de estudio \\
\hline Baños y cols. & 2008 & $\mathrm{RV}($ El mundo de EMMA) & Estudio de caso \\
\hline Andreu-Mateu & 2011 & $\mathrm{RV}($ El mundo de EMMA) & Estudio entre-grupos \\
\hline $\begin{array}{l}\text { Baños, Guillén, Quero, } \\
\begin{array}{l}\text { García-Palacios y Bote- } \\
\text { Ila }\end{array}\end{array}$ & 2011 & $\mathrm{RV}$ (EI mundo de EMMA) & Estudio entre-grupos \\
\hline $\begin{array}{l}\text { Andreu-Mateu, Botella, } \\
\text { Quero, Guillén y Baños }\end{array}$ & 2012 & $\mathrm{RV}$ (EI mundo de EMMA) & Estudio de caso \\
\hline
\end{tabular}




\begin{tabular}{lccc}
\hline \multicolumn{1}{c}{ Autores } & Año & TIC & Tipo de estudio \\
\hline $\begin{array}{l}\text { Quero, Molés, Pérez- } \\
\text { Ara, Botella y Baños }\end{array}$ & 2012 & Internet (TEO) & Estudio de caso \\
\hline $\begin{array}{l}\text { Quero, Botella, Andreu- } \\
\text { Mateu, Baños y Molés }\end{array}$ & 2013 & RV (El mundo de EMMA) & Estudio entre-grupos \\
\hline Molés y cols. & 2014 & Internet (TEO) & Series de casos \\
\hline Quero y cols. & En prensa & RV (El mundo de EMMA) & Serie de casos \\
\hline $\begin{array}{l}\text { Quero, Molés, Andreu- } \\
\text { Mateu, Baños y Botella }\end{array}$ & Sometido & RV (El mundo de EMMA) & Estudio entre-grupos \\
\hline
\end{tabular}

\section{Discusión}

Tras llevar a cabo la revisión de los tratamientos psicológicos, encontramos que, en primer lugar, solo hay recomendaciones generales, algunos estudios con sugerencias y estudios de investigación aislados que utilizan aproximaciones terapéuticas muy diversas.

Por una parte, los estudios no controlados (estudios de caso y series de casos) muestran deficiencias a la validez externa o generalización de los resultados que conllevan. Por otra parte, los pocos estudios experimentales hallados presentan importantes limitaciones metodológicas. En este sentido, algunos estudios no cuentan con descripciones detalladas de la muestra y utilizan grupos control inadecuados (por ejemplo, Gónzalez-Jaimes y Turnbull-Plaza, 2003), otros estudios se ven afectados por las altas tasas de abandono de los participantes (Hsiao y cols., 2012) o persiguen un objetivo diferente a la hora de realizar la investigación, como limitar la duración de las bajas médicas en las empresas (van der Klink y cols., 2003). De la misma manera, algunos de los abordajes utilizados se han centrado en aplicar estrategias de intervención quizá demasiado específicas como la meditación (Srivastava y cols., 2011). No obstante, intervenciones como la de Steinhardt y Dolbier (2008) o Ben-Itzhak y cols. (2012) se aproximan más a la base del trastorno, la dificultad de adaptación, aunque también cuentan con limitaciones metodológicas como la utilización de una muestra subclínica o la tasa significativa de abandono por parte de los participantes que repercute a la durabilidad de los logros de la terapia. Por otra parte, aquellos estudios que hacen uso de las TIC, concretamente la realidad virtual (EI mundo de EMMA) y el uso de internet (TEO), presentan resultados muy prometedores. No obstante, también presentan algunas limitaciones metodológicas ya que, o bien se trata de estudios no controlados, o los estudios experimentales realizados cuentan con una muestra reducida, el seguimiento incluido es muy corto o el número de técnicas incluidas en el protocolo para el TA de Botella y cols. (2008) hace difícil separar los efectos atribuibles a la RV del resto de estrategias. Sin embargo, ambos sistemas están resultando ser favorables y eficaces en el tratamiento de personas que padecen de TA.

Por lo tanto, podemos concluir que, a pesar de la alta prevalencia hallada en la literatura, del gran sufrimiento personal que causa y del deterioro significativo del funcionamiento a nivel social y laboral, todavía se hace presente la notable falta de estudios que se centren en los tratamientos del TA, no estando disponibles TPBE para este problema, por lo que la elección del tratamiento sigue siendo una decisión clínica. A la vista de los resultados obtenidos hasta el momento, en cuanto a futuras líneas de investigación, puede resultar favorable seguir apostando por las TIC como herramientas útiles en el tratamiento acompañadas de la TCC ya que 
los resultados obtenidos hasta la fecha han resultado ser prometedores y alentadores poniendo de manifiesto los beneficios y ventajas que tienen estos sistemas. Además, resultan de gran utilidad para dispensar o mejorar la aplicación de servicios terapéuticos logrando mejoras en los TPBE ya existentes.

\section{Referencias bibliográficas}

American Psychiatric Association (APA) (1952). Diagnostic and Statistical MANUAL of MeNTAL DISORDERS (DSM). Washington, DC: American Psychological Association.

American Psychiatric Association. (2003). En APA (2000). DIAGNOSTIC AND STATISTICAL MANUAL OF MENTAL DISORDERS DSM (4th ed., text revision). Washington, DC: American Psychological Association.

American Psychiatric Association (APA) (2013). DIAGNOSTIC AND STATISTICAL MANUAL OF MENTAL DISORDERS, 5. WASHINGTON, DC: American Psychological Association.

Andreasen, N. C. y Hoenck, P. (1982). The predictive value of adjustment disorders: a followup. American Journal of Psychiatry, 139, 584-590.

Andreu Mateu, M. S. (2011). Un protocolo de tratamiento psicológico para los Trastornos Adaptativos apoyado por un sistema de Realidad Virtual: análisis de su eficacia a través de un estudio controlado. Tesis Doctoral. Universitat Jaume I, Castellón de la Plana, España.

Andreu-Mateu, M. S., Botella, C., Quero, S., Guillén, V. y Baños, R. M. (2012). La utilización de la realidad virtual y estrategias de psicología positiva en el tratamiento de los trastornos adaptativos. Psicología conductual, 20, 323-348.

Andrews, A. (1995). Treatment outlines for the management of anxiety status: The quality assurance project. Australian and New Zealand Journal of Psychiatry, 19, 138-151.

Baños, R. M., Botella, C., Guillen, V., García-Palacios, A., Jorquera, M. y Quero, S. (2008). Un programa de tratamiento para los trastornos adaptativos. Un estudio de caso. Apuntes de Psicología, 26, 303-316.

Baños, R. M., Guillén, V., Quero, S., García-Palacios, A. y Botella, C. (2011). A virtual reality system for the treatment of stress-related disorders: A preliminary analysis of efficacy compared to a standard cognitive. International Journal of Human-Computer Studies, 69, 602-613.

Barraza, P. A. (2004). Evaluación e intervención psicológica en un caso de trastorno adaptativo mixto con ansiedad y estado de ánimo depresivo en una mujer con recidiva por cáncer de mama. Psicooncología: investigación y clínica biopsicosocial en oncología, 1 , 165-178.

Belchi, I., Vilardaga, R. y Bados, A. (2002). Tratamiento cognitivo-conductual de una persona con trastorno adaptativo en espera de un trasplante de pulmones. Cuadernos de Medicina Psicosomática y Psiquiátrica de Enlace, 62, 43-50.

Ben-Itzhak, S., Bluvstein, I., Schreiber, S., Aharonov-Zaig, I., Maor, M., Lipnik, R. y Bloch, M. (2012). The effectiveness of brief versus intermediate duration psychodynamic psychotherapy in the treatment of adjustment disorder. Journal of Contemporary Psychotherapy, 42, 249-256.

Benton, T. D. y Lynch, J. (2006). EMedicine. Adjustment Disorders. Extraído el 7 de junio de 2015 de http://www.emedicine.com/Med/topic3348.htm.

Botella, C., Baños, R. y Guillén, V. (2008). Creciendo en la adversidad. Una propuesta de tratamiento para los trastornos adaptativos. En C. Vázquez y G. Hervás (eds.), Psicología Positiva aplicada (pp. 129-154). Bilbao. Desclée de Brouwer. 
Casey, P. (2006). The 'afterthought' diagnosis: rehabilitating adjustment disorders. Expert Review of Neurotherapeutics, 6, 145-151. doi:10.1586/14737175.6.2.145.

Casey, P. (2009). Adjustment Disorder: epidemiology, diagnosis and treatment. CNS Drugs, 23, 927-938.

Casey, P. y Bailey, S. (2011). Adjustment disorders: the state of the art. World Psychiatry, 10, 11-18.

Despland, J. N., Monod, L. y Ferrero, F. (1995). Clinical relevance of adjustment disorder in DSM-III-R and DSM-IV. Comprehensive psychiatry,36, 454-460.

Fabrega, H., Mezzich, J. E. y Mezzich, A. C. (1987). Adjustment disorder as a marginal or transitional illness category in DSM-III. Archives of General Psychiatry, 44, 567-572.

Frankel, M. (2001). Ego enhancing treatment of adjustment disorders of later life. Journal of Geriatric Psychiatry, 34, 221-232.

González-Jaimes, E. I. y Turnbull-Plaza, B. (2003). Selection of psychotherapeutic treatment for adjustment disorder with depressive mood due to acute myocardial infarction. Archives of medical research, 34, 298-304.

Herrero, V. y Cano-Vindel, A. (2010). Un caso de trastorno adaptativo con ansiedad: evaluación, tratamiento y seguimiento. Anuario de Psicología Clínica y de la Salud, 6, 53-59.

Hsiao, F. H., Lai, Y. M., Chen, Y. T., Yang, T. T., Liao, S. C., Ho, R. T. y cols. (2014). Efficacy of psychotherapy on diurnal cortisol patterns and suicidal ideation in adjustment disorder with depressed mood. General Hospital Psychiatry, 36, 214-219.

Jojić, B. R. y Leposavić, L. M. (2005). Autogenic training as a therapy for adjustment disorder in adolescents. Srpski arhiv za celokupno lekarstvo,133, 424-428.

Maercker, A., Einsle, F. y Köllner, V. (2007). Adjustment disorders as stress response syndromes: a new diagnostic concept and its exploration in a medical sample. Psychopathology, 40, 135-146.

Mihelich (2000). Eye movement desensitization and reprocessing treatment of adjustment disorder. University of Tulsa. Extraído el 19 de enero de http://emdria.omeka.net/items/ show/17459.

Molés, M., Quero, S., Nebot, S., Soler, C., Campos, D. y Botella, C. An Online Emotional Therapy System (TEO) to Deliver Homework Assignments in the Treatment of Adjustment Disorders versus the Traditional Method (Octubre, 2014). Ponencia presentada en 7th ISRII Scientific Meeting. Valencia (España).

Nardi, C., Lichtenberg, P. y Kaplan, Z. (1994). Adjustment disorder of conscripts as a military phobia. Military Medicine, 159, 612-616.

Powell, S. y McCone, D. (2004). Treatment of adjustment disorder with anxiety: A September 11, 2001, case study with a 1-year follow-up. Cognitive and Behavioral Practice, 11, 331336.

Quero, S., Andreu-Mateu, S., Moragrega, I., Baños, R. M., Molés, M., Nebot, S. y Botella, C. (en prensa). Un Programa Cognitivo-Conductual que utiliza la Realidad Virtual para el Tratamiento de los Trastornos Adaptativos: Una Serie de casos. Revista Argentina de Psicología Clínica.

Quero, S., Botella C., Andreu-Mateu, S., Baños R. M. y Molés, M. (2013). An Adaptive Virtual Reality System for the Treatment of Adjustment Disorder: 12-month Follow-up Efficacy data. ABCT's 47th Annual Convention. Nashville, TN.

Quero, S., Molés, M., Pérez-Ara, M. A., Botella, C. y Baños, R. M. (2012). An Online Emotional Regulation System to deliver homework assignments for treating Adjustment Disorders. Journal of CyberTherapy \& Rehabilitation, 5, 23-24.

Silverstone, PH. (1996). Prevalence of psychiatric disorders in medical in-patients. Journal of Nervous Mental Disease, 184, 43-51. 
Srivastava, M., Talukdar, U. y Lahan, V. (2011). Meditation for the management of adjustment disorder anxiety and depression. Complementary therapies in clinical practice, 17, 241245.

Steinhardt, M. y Dolbier, C. (2008). Evaluation of a resilience intervention to enhance coping strategies and protective factors and decrease symptomatology. Journal of American College Health, 56, 445-453.

Strain, J. J. (1995). Adjustment disorders. En G. O. Gabbard (ed.), Treatments of Psychiatric Disorders (2. ${ }^{a}$ ed.) (pp. 1656-1665). Washington, DC: APA press.

Strain, J. J. y Diefenbacher, A. (2008). The adjustment disorders: the conundrums of the diagnoses. Comprehensive Psychiatry, 49, 121-130.

Strain, J. J., Klipstein, K. G. y Newcorn, J. H. (2010). Adjustment disorders. En Hales, Yudofsky y Gabbard (eds.), Essentials of Psychiatry (pp. 255-269). Arlington: American Psychiatric Publishing.

Strain, J. J., Smith, G. C., Hammer, J. S., McKenzie, D. P., Blumenfield, M., Muskin, P. y cols. (1998). Adjustment disorder: a multisite study of its utilization and interventions in the consultation-liaison psychiatry setting. General hospital psychiatry, 20, 139-149.

Taggart, C., O'Grady, J., Stevenson, M. y cols. (2006). Accuracy of diagnosis and routine psychiatric assessment in patients presenting to an accident and emergency department. General Hospital Psychiatry, 8, 330-335.

Van der Klink, J. L., Blonk, R. W., Schene, A. H. y van Dijk, F. J. (2003). Reducing long term sickness absence by an activating intervention in adjustment disorders. Occupational and Environmental Medicine, 60, 429-437. 